\title{
The static potential from the selected intermediate states of gluons
}

\author{
Yoshiaki Koma*t \\ Numazu College of Technology \\ E-mail: koma@numazu-ct.ac.jp \\ Miho Koma \\ Nihon University, College of International Relations \\ E-mail: koma.miho@nihon-u.ac.jp
}

\begin{abstract}
By employing the multilevel algorithm, we investigate the static inter-quark potential from the Polyakov loop correlation function (PLCF) constructed from the selected intermediate states of gluons. While the use of partial intermediate states cannot guarantee gauge invariance of the PLCF, we find that the functional forms of the PLCF and then the static potential can be unchanged from the gauge invariant ones, which indicates that all possible intermediate states equally contribute to the nonperturbative potential. We demonstrate this not only in the quarkantiquark system but also in the three-quark system.
\end{abstract}

The 31st International Symposium on Lattice Field Theory

July 29 - August 3, 2013

Mainz, Germany

*Speaker.

$\dagger$ Y.K. is partially supported by the Ministry of Education, Science, Sports and Culture, Japan, Grant-in-Aid for Young Scientists (B) (24740176). The main calculation has been performed on the NEC SX8 at Research Center for Nuclear Physics (RCNP), Osaka University, Japan. 


\section{Introduction}

The static inter-quark potential reflects a nontrivial structure of the QCD vacuum. The lattice QCD simulations offer nonperturbative computation of the potential, and it is well-known that the quark-antiquark potential at zero temperature exhibits a linear rising behavior as a function of the quark-antiquark distance, which means that it is impossible to isolate a single quark with finite energy and thus quarks are confined. The precise determination of the potential at long distances is of interest for various purposes; clarifying the mechanism of quark confinement, investigating the correspondence to the QCD string theory, applying to the heavy quarkonium spectroscopy, and so on. Usually the expectation value of the Wilson loop is computed to extract the potential, but as explained below, whoever computes the potential at long distances is required to adopt smearing techniques or alternative methods to the operators, which may cause systematic errors.

In this paper, we present some results on the static potential obtained by computing the Polyakov loop correlation function (PLCF) instead of the Wilson loop with the help of the multilevel algorithm [1, 2]. The PLCF in the quark-antiquark system is defined by a pair of spatially separated Polyakov and anti-Polyakov loops, which is, as explained below, easy to extract the ground state potential once the PLCF is determined accurately. We find that the use of the multilevel algorithm also allows us to compute the PLCF with the selected intermediate states of gluons; while the use of partial intermediate states cannot guarantee apparent gauge invariance of the PLCF, the functional form of the PLCF and then the static potential can be unchanged from the gauge invariant ones up to calculable weight factors, which indicates that all possible intermediate states equally contribute to the nonperturbative potential. We also find that the situation is the same in the three-quark system.

\section{The Wilson loop vs. the PLCF for the static quark-antiquark potential}

We consider SU(3) lattice gauge theory in four dimensions with the lattice volume $L^{3} \times T$ and the lattice spacing $a$, and impose periodic boundary conditions in all space-time directions.

The heavy quark potential is usually computed from the expectation value of the Wilson loop, the trace of the path-ordered product of link variables $U_{\mu}(x)$, which is also expressed as

$$
W(r, t)=\mathbb{L}\left(x_{0}=0, \vec{x}_{1}, \vec{x}_{2}\right)_{\alpha \gamma}^{*}\left\{\mathbb{T}\left(0, \vec{x}_{1}, \vec{x}_{2}\right) \mathbb{T}\left(a, \vec{x}_{1}, \vec{x}_{2}\right) \cdots \mathbb{T}\left(t-a, \vec{x}_{1}, \vec{x}_{2}\right)\right\}_{\alpha \beta \gamma \delta} \mathbb{L}\left(x_{0}=t, \vec{x}_{1}, \vec{x}_{2}\right)_{\beta \delta},
$$

where Greek indices take the values form 1 to 3 in the SU(3) case and repeated indices are assumed to be summed over. $\mathbb{L}\left(0, \vec{x}_{1}, \vec{x}_{2}\right)_{\alpha \gamma}^{*}$ and $\mathbb{L}\left(t, \vec{x}_{1}, \vec{x}_{2}\right)_{\beta \delta}$ are products of the spatial link variables from $\vec{x}_{1}$ to $\vec{x}_{2}$ at $x_{0}=0$ and $t$, corresponding to the quark source and sink, respectively.

$$
\mathbb{T}\left(x_{0}, \vec{x}_{1}, \vec{x}_{2}\right)_{\alpha \beta \gamma \delta} \equiv U_{4}\left(x_{0}, \vec{x}_{1}\right)_{\alpha \beta} U_{4}\left(x_{0}, \vec{x}_{2}\right)_{\gamma \delta}^{*}
$$

is the two-link correlator, a direct product of two temporal link variables separated by a distance $r=\left|\vec{x}_{1}-\vec{x}_{2}\right|$, which consists of a $9 \times 9$ complex matrix. The two-link correlator acts on the color states in the $\mathbf{3} \otimes \overline{\mathbf{3}}$ representation of the $\mathrm{SU}(3)$ group $\left|n ; \vec{x}_{1}, \vec{x}_{2}\right\rangle_{\alpha \beta}$, which is the eigenstate of the hamiltonian $\mathbb{H}$ defined by the transfer matrix in the temporal gauge, $\mathbb{T} \equiv e^{-\mathbb{H} a}$, and then satisfies $\mathbb{T}\left(x_{0}, \vec{x}_{1}, \vec{x}_{2}\right)_{\alpha \lambda \gamma \varepsilon}\left|n ; \vec{x}_{1}, \vec{x}_{2}\right\rangle_{\alpha \gamma}=e^{-E_{n}(r) a}\left|n ; \vec{x}_{1}, \vec{x}_{2}\right\rangle_{\lambda \varepsilon}$, where $n$ is the principal quantum number. The energies $E_{n}(r)$ are positive, which should be common to all color components of $\left|n ; \vec{x}_{1}, \vec{x}_{2}\right\rangle_{\alpha \beta}$. The 


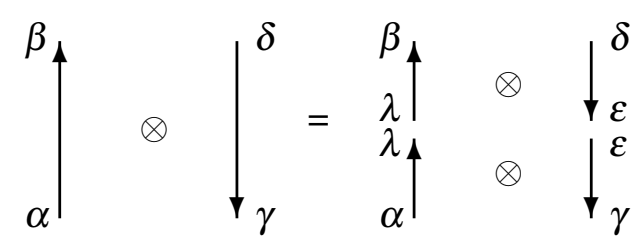

Figure 1: A product of two-link correlators, $\left\{\mathbb{T}\left(x_{0}, \vec{x}_{1}, \vec{x}_{2}\right) \mathbb{T}\left(x_{0}+a, \vec{x}_{1}, \vec{x}_{2}\right)\right\}_{\alpha \beta \gamma \delta}=\mathbb{T}\left(x_{0}, \vec{x}_{1}, \vec{x}_{2}\right)_{\alpha \lambda \gamma \varepsilon} \mathbb{T}\left(x_{0}+\right.$ $\left.a, \vec{x}_{1}, \vec{x}_{2}\right)_{\lambda \beta \varepsilon \delta}$.

multiplication law of the two-link correlators between two neighboring time slices $x_{0}$ and $x_{0}+a$ (see Fig. 10 for a schematic understanding) is

$$
\left\{\mathbb{T}\left(x_{0}, \vec{x}_{1}, \vec{x}_{2}\right) \mathbb{T}\left(x_{0}+a, \vec{x}_{1}, \vec{x}_{2}\right)\right\}_{\alpha \beta \gamma \delta}=\mathbb{T}\left(x_{0}, \vec{x}_{1}, \vec{x}_{2}\right)_{\alpha \lambda \gamma \varepsilon} \mathbb{T}\left(x_{0}+a, \vec{x}_{1}, \vec{x}_{2}\right)_{\lambda \beta \varepsilon \delta} .
$$

Based on the transfer matrix formalism, the expectation value of the Wilson loop is evaluated by inserting the complete set of eigenstates $1=\sum_{m=0}^{\infty}\left|m ; \vec{x}_{1}, \vec{x}_{2}\right\rangle\left\langle m ; \vec{x}_{1}, \vec{x}_{2}\right|$ at time slices $x_{0}=a, 2 a, \ldots, t-$ $a$ as

$$
\langle W(r, t)\rangle=\sum_{n=0}^{\infty} w_{n}(r, t) e^{-E_{n}(r) t}
$$

where $w_{n}(r, t)=\left\langle 0\left|\mathbb{L}\left(0, \vec{x}_{1}, \vec{x}_{2}\right)^{*}\right| n ; \vec{x}_{1}, \vec{x}_{2}\right\rangle\left\langle n ; \vec{x}_{1}, \vec{x}_{2}\left|\mathbb{L}\left(t, \vec{x}_{1}, \vec{x}_{2}\right)\right| 0\right\rangle$ and $|0\rangle$ denotes the vacuum. If we pay attention to the ground state energy $E_{0}(r)$, which is regarded as the static potential $V(r)$, Eq. (2.4) is written as

$$
-\frac{1}{t} \ln \langle W(r, t)\rangle=E_{0}(r)-\frac{1}{t} \ln w_{0}(r, t)+O\left(\frac{1}{t} \frac{w_{1}}{w_{0}} e^{-\left(E_{1}-E_{0}\right) t}\right),
$$

and thus $V(r) \equiv E_{0}(r)=-\lim _{t \rightarrow \infty} \frac{1}{t} \ln \langle W(r, t)\rangle$. One may find that it is not straightforward to extract the ground state potential from Eq. (2.5) due to the unknown weight $w_{0}$ and contamination from the excited states because $t$ cannot be large practically with the limitation $t<T / 2$. In order to suppress the unwanted second and third terms in Eq. 2.5], one then adopts smearing techniques for better overlap with the ground state to achieve $w_{0}(r, t) \rightarrow 1$. Otherwise it requires high simulation cost to perform simulations with large $T$.

The heavy quark potential may also be extracted from the PLCF. Using the two-link correlators, the operator for the PLCF is constructed as

$$
\operatorname{Tr} P\left(\vec{x}_{1}\right) \operatorname{Tr} P\left(\vec{x}_{2}\right)^{*}=\left\{\mathbb{T}\left(0, \vec{x}_{1}, \vec{x}_{2}\right) \mathbb{T}\left(a, \vec{x}_{1}, \vec{x}_{2}\right) \cdots \mathbb{T}\left(T-a, \vec{x}_{1}, \vec{x}_{2}\right)\right\} \alpha \alpha \gamma \gamma
$$

The expectation value is evaluated by inserting the complete set of eigenstates at all time slices $x_{0}=0, a, \ldots, T-a$ as

$$
\left\langle\operatorname{Tr} P\left(\vec{x}_{1}\right) \operatorname{Tr} P\left(\vec{x}_{2}\right)^{*}\right\rangle=\sum_{n=0}^{\infty} w_{n} e^{-E_{n}(r) T},
$$

where $w_{0}=1$ is guaranteed by construction, which is one of the main difference from the Wilson loop. We thus find

$$
-\frac{1}{T} \ln \left\langle\operatorname{Tr} P\left(\vec{x}_{1}\right) \operatorname{Tr} P\left(\vec{x}_{2}\right)^{*}\right\rangle=E_{0}(r)+O\left(\frac{1}{T} e^{-\left(E_{1}-E_{0}\right) T}\right),
$$


and immediately obtain the ground state potential by $V(r) \equiv E_{0}(r)=-\frac{1}{T} \ln \left\langle\operatorname{Tr} P\left(\vec{x}_{1}\right) \operatorname{Tr} P\left(\vec{x}_{2}\right)^{*}\right\rangle$, since the error terms of $O\left(\frac{1}{T} e^{-\left(E_{1}-E_{0}\right) T}\right)$ are already negligible at zero temperature. This construction is theoretically clean and the extraction of the potential seems to be quite easy. However, the problem is that it is impossible to compute the PLCF accurately within ordinary simulations as the expectation values are extremely small at long distances, which are easily obscured by the statistical noise.

\section{The PLCF with the multilevel algorithm}

Using the multilevel algorithm, it is possible to overcome the problem of the smallness of the PLCF. The idea of the multilevel algorithm is to compute the desired correlation function, which may have extremely small expectation value, from the product of relatively large sublattice average of its components, where the sublattices are defined by dividing the lattice volume into several layers along the time direction. During the computation of the sublattice averages, the spatial links at the sublattice boundaries are fixed. The computation of the correlation function in this way is supported by the transfer matrix formalism and is regarded as the hierarchical functional integral method. It has already been applied to computing not only the original PLCF [1, 2] but also the glueball spectrum [3 4], the flux-tube profile [5], and the relativistic corrections to the quark-antiquark potential (field strength correlators) [6,7, and so on.

Let us briefly explain what is done in the multilevel algorithm when computing the PLCF. As an example, we consider a simple case that the lattice volume is divided into two sublattices $\left(N_{\text {sub }}=2\right)$ at the time slice $x_{0}=0$ and $x_{0}=T / 2$. The number of the time slices in a sublattice is $N_{\text {tsl }}=T /\left(a N_{\text {sub }}\right)$. We then compute the averages of $\left\{\mathbb{T}\left(0, \vec{x}_{1}, \vec{x}_{2}\right) \cdots \mathbb{T}\left(T / 2-a, \vec{x}_{1}, \vec{x}_{2}\right)\right\}_{\alpha \lambda \gamma \varepsilon}$ and $\left\{\mathbb{T}\left(T / 2, \vec{x}_{1}, \vec{x}_{2}\right) \cdots \mathbb{T}\left(T-a, \vec{x}_{1}, \vec{x}_{2}\right)\right\}_{\lambda \beta \varepsilon \delta}$ and construct the final operator by

$$
\operatorname{Tr} P\left(\vec{x}_{1}\right) \operatorname{Tr} P\left(\vec{x}_{2}\right)^{*}=\left[\mathbb{T}\left(0, \vec{x}_{1}, \vec{x}_{2}\right) \cdots \mathbb{T}\left(\frac{T}{2}-a, \vec{x}_{1}, \vec{x}_{2}\right)\right]_{\alpha \lambda \gamma \varepsilon}\left[\mathbb{T}\left(\frac{T}{2}, \vec{x}_{1}, \vec{x}_{2}\right) \cdots \mathbb{T}\left(T-a, \vec{x}_{1}, \vec{x}_{2}\right)\right]_{\lambda \alpha \varepsilon \gamma},
$$

where $[\cdots]$ represents taking the sublattice average. Note that fixing the spatial links at the sublattice boundaries correspond to inserting two normalized fixed sources $\left|\phi_{1}\right\rangle_{\alpha \beta}=\sum_{n=0}^{\infty} a_{n}\left|n ; \vec{x}_{1}, \vec{x}_{2}\right\rangle_{\alpha \beta}$ and $\left|\phi_{2}\right\rangle_{\alpha \beta}=\sum_{m=0}^{\infty} b_{m}\left|m ; \vec{x}_{1}, \vec{x}_{2}\right\rangle_{\alpha \beta}$ at $x_{0}=0$ and $x_{0}=T / 2$, respectively, where $a_{n}$ and $b_{m}$ are unknown a priori but satisfy $\sum_{n=0}^{\infty}\left|a_{n}\right|^{2}=\sum_{n=0}^{\infty}\left|b_{n}\right|^{2}=1$. Then, Eq. (3.1) becomes

$$
\begin{aligned}
\operatorname{Tr} P\left(\vec{x}_{1}\right) \operatorname{Tr} P\left(\vec{x}_{2}\right)^{*} & =\operatorname{Tr}\left[\left\langle\phi_{1}\left|\mathbb{T}(0) \cdots \mathbb{T}\left(\frac{T}{2}-a\right)\right| \phi_{2}\right\rangle\right]\left[\left\langle\phi_{2}\left|\mathbb{T}\left(\frac{T}{2}\right) \cdots \mathbb{T}(T-a)\right| \phi_{1}\right\rangle\right] \\
& =\sum_{\alpha \gamma} \sum_{\lambda \varepsilon}\left(\sum_{n=0}^{\infty} a_{n}^{*} b_{n} e^{-E_{n}(r)(T / 2)} \cdot \sum_{m=0}^{\infty} b_{m}^{*} a_{m} e^{-E_{m}(r)(T / 2)}\right) .
\end{aligned}
$$

If we take the average for different fixed sources at $x_{0}=0$ and $x_{0}=T / 2$ of other independent configurations, we obtain $\left\langle\operatorname{Tr} P\left(\vec{x}_{1}\right) \operatorname{Tr} P\left(\vec{x}_{2}\right)^{*}\right\rangle$ as in Eq. 2.77, since inserting the fixed sources finally reduces to inserting the complete set.

At this point, it is interesting to notice that if $T / 2$ is large enough such that the terms of $O\left(e^{-\left(E_{1}-E_{0}\right)(T / 2)}\right)$ are negligible, which is usually the case at zero temperature, Eq. (3.2) further reduces to

$$
\operatorname{Tr} P\left(\vec{x}_{1}\right) \operatorname{Tr} P\left(\vec{x}_{2}\right)^{*}=\sum_{\alpha \gamma} \sum_{\lambda \varepsilon} \underbrace{\left|a_{0}\right|^{2}\left|b_{0}\right|^{2} e^{-E_{0}(r) T}}_{\text {independent of } \alpha \gamma \lambda \varepsilon}=3^{4}\left|a_{0}\right|^{2}\left|b_{0}\right|^{2} e^{-E_{0}(r) T} .
$$


In this case, the ground state potential $E_{0}(r)$ can be extracted even from "one" configuration. Moreover, Eq. (3.3) reads that each color component of the intermediate states "equally" contributes to the exponential decay of the PLCF, which means that it is possible to extract the gauge-invariant potential from the gauge-variant PLCF with selected partial intermediate states.

Note that if the terms of $O\left(e^{-\left(E_{1}-E_{0}\right)(T / 2)}\right)$ in Eq. (3.2) are not negligible, one has to use large temporal lattice volume from the beginning. When $T$ is large enough, it is possible to start from smaller temporal size of the sublattices (choose $N_{\text {sub }}>2$ for $N_{\text {tsl }}=T / a N_{\text {sub }}$ ). In any case, if the ground state energy is of interest, the sublattice averaging must be carried out up to higher levels until the terms of $O\left(e^{-\left(E_{1}-E_{0}\right)\left(T / N_{\text {sub }}\right)}\right)$ become negligible. Otherwise the contamination from the excited states cannot be avoided. It means that there is a critical minimal size of $a N_{\text {tsl }}$ to obtain the ground state energy. In our experience with the Wilson gauge action, we find that $a N_{\text {tsl }} \simeq 0.38 \mathrm{fm}$ is the minimal size, corresponding to $N_{\mathrm{tsl}}=3$ at $\beta=5.8, N_{\mathrm{tsl}}=4$ at $\beta=6.0$, and $N_{\mathrm{tsl}}=6$ at $\beta=6.2$.

\section{Numerical results}

Using the multilevel algorithm, we shall demonstrate that the PLCF and the potential can be computed from one configuration with the gauge-variant PLCF constructed from the selected intermediate states. We stick to the Wilson gauge action at $\beta=6.0$ and then set $N_{\mathrm{tsl}}=4$.

The first result is shown in Fig. 2, which consists of the PLCF in the quark-antiquark system on the $24^{4}$ lattice ( $N_{\text {sub }}=24 / 4=6$ ) as a function of the number of internal sublattice updates $N_{\text {iupd }}$ (left) and the corresponding potential (right). We have computed two types of the PLCF; one is the ordinary gauge-invariant PLCF (denoted as "full") and the other is the gauge-variant PLCF constructed from the diagonal components of the sublattice correlators (denoted as "diagonal"). The expected weight factors for these PLCFs are, full : diagonal $=\left(3^{N_{\text {sub }}-1}\right)^{2}: 1=59049: 1$. Therefore we have multiplied the factor 59049 to the diagonal PLCF for a direct comparison. We find that both PLCFs reduce to the same values with increasing the $N_{\text {iupd }}$. The potentials are then computed from these PLCFs using the values of the largest number of $N_{\text {iupd }}$ (in this case $N_{\text {iupd }}=100000$ ), which clearly lead to the same result. Note that the potential plotted here is of one configuration, but the fitting result with the functional form $V(r)=-\alpha / r+\sigma r+\mu$ is consistent with that uses more independent configurations $[7]$.

The second result is shown in Fig. 3, which is similar to the first one but on the $16^{4}$ lattice $\left(N_{\text {sub }}=4\right)$ and we have computed three types of the PLCF; the ordinary gauge-invariant PLCF ("full"), the gauge-variant PLCFs which are constructed from the diagonal ("diagonal") and the off-diagonal ("off-diagonal") components of the two-link correlators, respectively. The expected weight factors for these PLCFs are, full : diagonal : off-diagonal $=\left(3^{T / a-1}\right)^{2}: 1:\left(\frac{2^{T / a}+2(-1)^{T / a}}{3}\right)^{2}=$ $205891132094649: 1: 477247716$. Therefore we have multiplied the factor 205891132094649 to the diagonal PLCF and 205891132094649/477247716 to the off-diagonal PLCF for a direct comparison. Again, we find that all PLCFs reduce to the same values with increasing the $N_{\text {iupd }}$. Although the diagonal PLCF requires relatively a large number of $N_{\text {iupd }}$, it is reasonable that the diagonal PLCF is 14 orders of magnitude smaller than the full PLCF. The potentials are then computed from these PLCFs using the values of the largest number of $N_{\text {iupd }}=3060000$ and are found to be exactly the same. The first and second results strongly support our expectation that it is possible to obtain the gauge-invariant PLCF and the static potential with the selected intermediate states. 

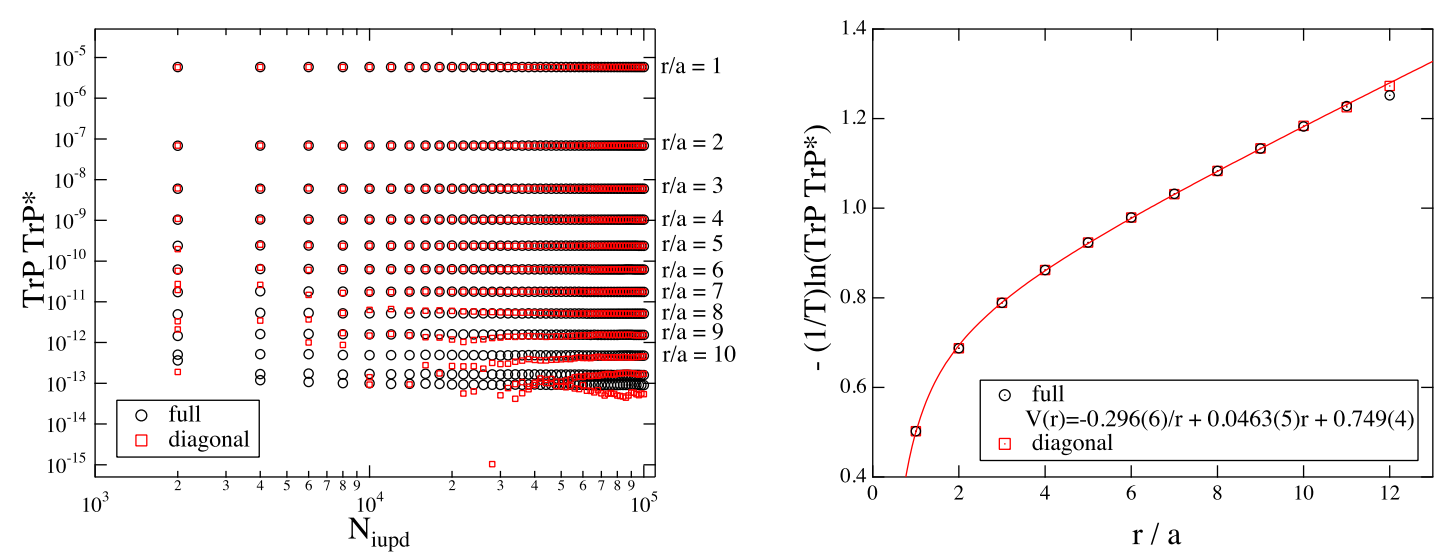

Figure 2: The PLCF (left) and the potential (right) in the quark-antiquark system on the $24^{4}$ lattice $\left(N_{\text {sub }}=\right.$ $6)$.
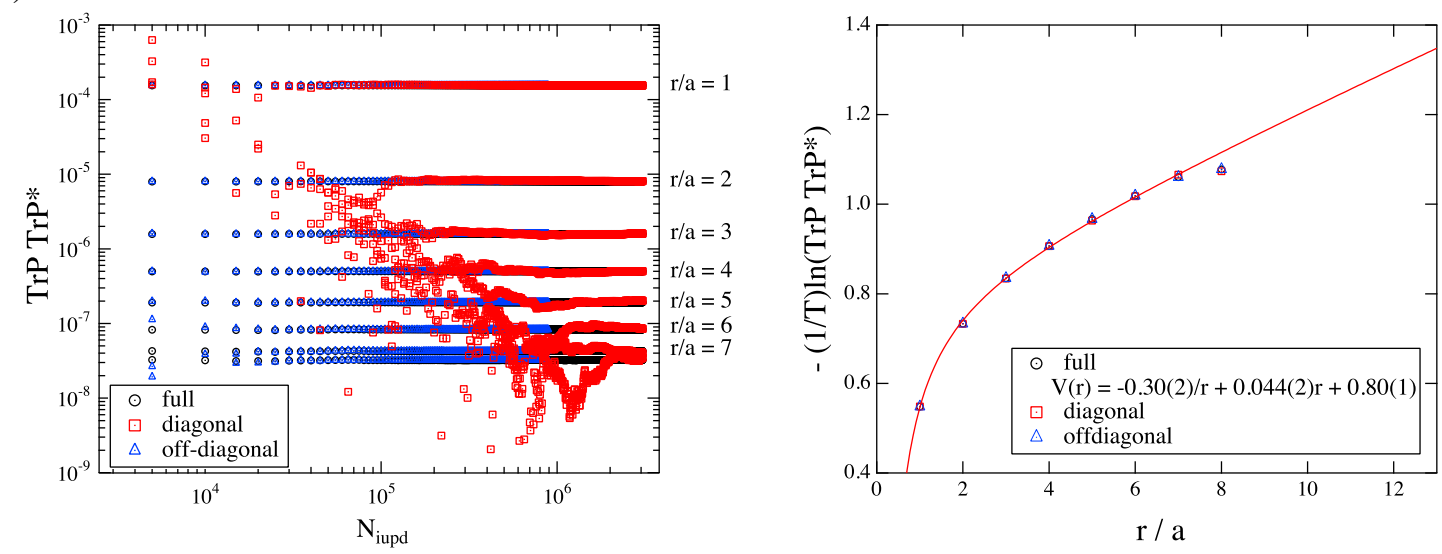

Figure 3: The PLCF (left) and the potential (right) in the quark-antiquark system on the $16^{4}$ lattice $\left(N_{\text {sub }}=\right.$ 4).

The final result is shown in Fig. 44 in which we have extended the above idea to the three-quark system. In this case, the PLCF consists of the "three-link correlators",

$$
\mathbb{T}\left(x_{0}, \vec{x}_{1}, \vec{x}_{2}, \vec{x}_{3}\right)_{\alpha \beta \gamma \delta \varepsilon \zeta} \equiv U_{4}\left(x_{0}, \vec{x}_{1}\right)_{\alpha \beta} U_{4}\left(x_{0}, \vec{x}_{2}\right)_{\gamma \delta} U_{4}\left(x_{0}, \vec{x}_{3}\right)_{\varepsilon \zeta}
$$

The multilevel algorithm can be applied in the same manner as in the quark-antiquark system, but the three-link correlator requires larger computer memory as it is composed of a $9 \times 9 \times 9$ complex matrix. We have put the three Polyakov loops at $\vec{x}_{1}=(r, 0,0), \vec{x}_{2}=(0, r, 0), \vec{x}_{3}=(0,0, r)$, respectively. Therefore, $r$ is not immediately regarded as the distance among quarks. We have computed the gauge-invariant PLCF ("full") and the gauge-variant PLCF with the diagonal sublattice correlator ("diagonal"). The expected ratio is, full : diagonal $=\left(3^{N_{\text {sub }}-1}\right)^{3}: 1=14348907: 1$, and then we have multiplied the factor 14348907 to the diagonal PLCF. Although the diagonal PLCF is still fluctuating at long distances, we see such a behavior that both PLCFs reduce to the same values with increasing the $N_{\text {iupd }}$, which is quite the same as in the quark-antiquark system. The potentials are then computed from these PLCFs using the values of the largest number of $N_{\text {iupd }}=530000$. Our result, up to $r / a=4$, is comparable to the result of Takahashi et al. [8 9], which uses the 

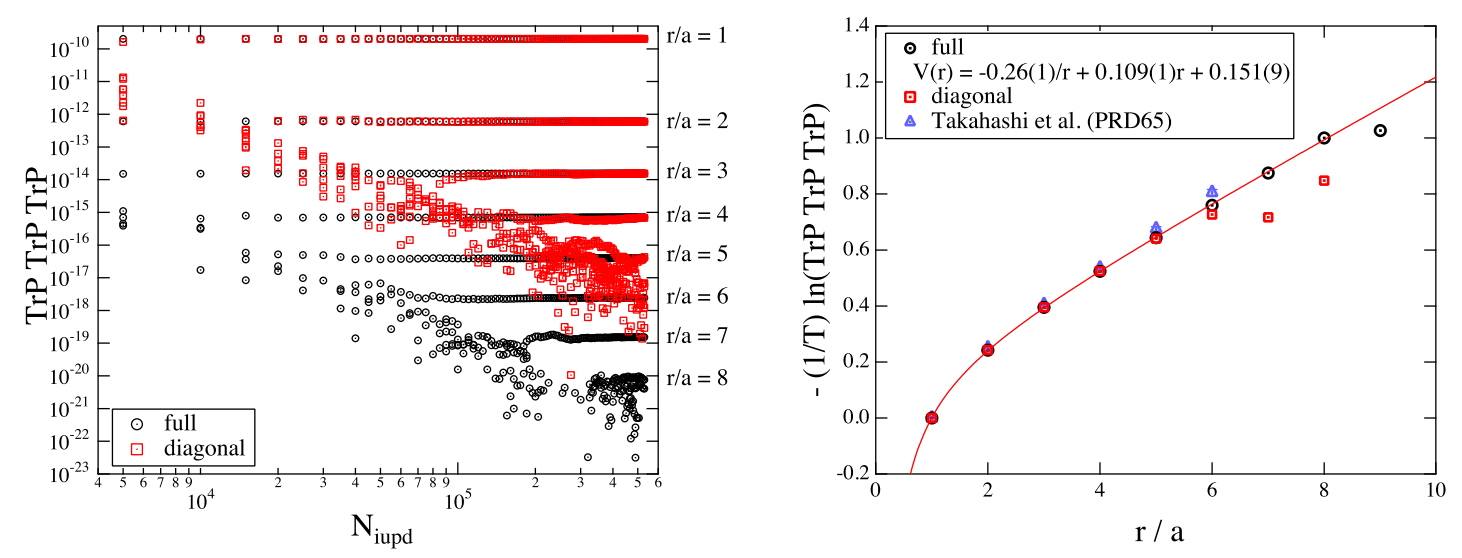

Figure 4: The PLCF (left) and the potential in the three-quark system on the $24^{4}$ lattice $\left(N_{\text {sub }}=6\right)$.

three-quark Wilson loop with the smearing techniques, where the both potentials are normalized at $r / a=1$. The small deviation at $r / a>5$ may be clarified by further simulations.

\section{Summary}

By employing the multilevel algorithm, we have investigated the static inter-quark potentials in the quark-antiquark and the three-quark systems from the PLCF constructed from the selected intermediate states. While the use of partial intermediate states cannot guarantee gauge invariance of the PLCF, we have found that the functional forms of the PLCF and the static potential are unchanged from the gauge invariant ones, which indicates that each color component of the intermediate states equally contributes to the PLCF. In other words, there is no dominant color component to the potential. In this sense, gauge invariance of the operator is desirable with respect to maximizing the number of internal color statistics, which may help to obtain stable signals even with the small number of internal sublattice updates. However, if the operator requires huge computer memory which cannot be simulated with available resources, the selection of the intermediate state can be a possible option from the point of view of saving the computer memory in the multilevel algorithm.

\section{References}

[1] M. Lüscher and P. Weisz, JHEP 0109 (2001) 010, hep-lat/0108014.

[2] M. Lüscher and P. Weisz, JHEP 0207 (2002) 049, hep-lat/0207003.

[3] H. B. Meyer, JHEP 0301 (2003) 048, hep-lat/0209145.

[4] P. Majumdar, Y. Koma, and M. Koma, Nucl.Phys. B677 (2004) 273-288, hep-lat/0309003.

[5] Y. Koma, M. Koma, and P. Majumdar, Nucl.Phys. B692 (2004) 209-231, hep-lat/0311016.

[6] Y. Koma, M. Koma, and H. Wittig, Phys.Rev.Lett. 97 (2006) 122003, hep-lat/0607009.

[7] Y. Koma and M. Koma, Nucl.Phys. B769 (2007) 79-107, hep-lat/0609078.

[8] T. T. Takahashi, H. Matsufuru, Y. Nemoto, and H. Suganuma, Phys.Rev.Lett. 86 (2001) 18-21, hep-lat/0006005.

[9] T. T. Takahashi, H. Suganuma, Y. Nemoto, and H. Matsufuru, Phys.Rev. D65 (2002) 114509, hep-lat/0204011. 